\title{
Construcción y validación de un instrumento para el análisis de la formación deportiva en voleibol
}

\section{Desing and validation of an instrument for analysis of sportive formation in volleyball}

\section{Construção e validação de um instrumento para a análise da formação esportiva em voleibol}

\author{
Collet, C. ${ }^{1}$, Nascimento, J. V. ${ }^{2}$, Folle, A. ${ }^{1}$, Ibáñez, S. J. ${ }^{3}$ \\ ${ }^{1}$ Universidade do Estado de Santa Catarina; ${ }^{2}$ Universidade Federal de Santa Catarina; ${ }^{3}$ Universidad de \\ Extremadura
}

\begin{abstract}
RESUMEN
El objetivo de este estudio fue construir y verificar la validez de contenido y la fiabilidad del Instrumento de Análisis de la Formación Deportiva en Voleibol (IAFDV). Participaron en el proceso de validez de contenido 12 jueces expertos que cumplieron los criterios de selección. Para verificar la fiabilidad (estabilidad y consistencia interna) participaron 15 jugadores de categorías de formación de voleibol. La validez de contenido se analizó a partir de la $V$ Aiken, la estabilidad del instrumento se analizó a través del Coeficiente de Spearman y la consistencia interna mediante el coeficiente Alpha de Cronbach. Los resultados ponen de manifiesto los altos índices de validez de contenido (.91) y fiabilidad del instrumento, teniendo en cuenta que 77 de los 78 ítems presentaron una correlación significativa $(p<.05)$ y alta consistencia interna $(\alpha>.70)$. Así, se considera que el cuestionario es adecuado y tiene validez y fiabilidad satisfactorios para medir el proceso de formación deportiva de los jugadores de voleibol.
\end{abstract}

Palabras clave: Desarrollo deportivo. Elementos dinámicos. Etapas de la formación deportiva.

\begin{abstract}
The aim of this study was to develop and verify the content validity and reliability of the Sports Training Instrument in Volleyball (IAFDV). Participated in the content validity process 12 expert judges who met the selection criteria. In order to verify the reliability (stability and internal consistency) participated 15 players from volleyball training categories. The content validity was analysed from the $\mathrm{V}$ Aiken, the stability of the instrument was analysed through the Spearman coefficient and the internal consistency by means of the Cronbach's Alpha coefficient. The results show the high content validity indexes (.91) and reliability of the instrument, taking into account that 77 of the 78 items presented a significant correlation $(p<.05)$ and high internal consistency $(\alpha>.70)$. Thus, it is considered that the questionnaire is adequate and has satisfactory validity and reliability to measure the sports training process of volleyball players.
\end{abstract}

Keywords: Sports development. Dynamic elements. Stages of sports development.

Correspondence to: Carine Collet. Dirección Postal: Laboratório de

Pedagogia do Esporte. Campus Reitor João David Ferreira Lima.

Florianópolis, SC/Brasil.

Tel: +55 (48) 3721-8526. Email: ca_collet@hotmail.com 


\section{Construcción y validación de un instrumento para el análisis de la formación deportiva en voleibol}

\section{RESUMO}

O objetivo deste estudo foi construir e verificar a validade de conteúdo e a confiabilidade do Instrumento de Análise da Formação desportiva no Voleibol (IAFDV). Participaram no processo de validação de conteúdo 12 juízes especialistas que preencheram os critérios de seleção. Para verificar a confiabilidade (estabilidade e consistência interna) participaram 15 jogadores de categorias de formação do voleibol. A validade de conteúdo foi analisada a partir da V Aiken, a estabilidade do instrumento foi analisada através do coeficiente de Spearman e da consistência interna atravésdo coeficiente Alpha de Cronbach. Os resultados evidenciam altos índices de validade de conteúdo (.91) e a confiabilidade do instrumento, levando-se em conta que 77 dos 78 itens apresentaram correlação significativa $(\mathrm{p}<.05)$ e alta consistência interna $(\alpha>.70)$. Assim, considera-se que o questionário é adequado, possui validade e confiabilidade satisfatórias para avaliar o processo de formação desportiva de jogadores de voleibol.

Palavras chave: Desenvolvimento esportivo. Elementos dinâmicos. Estágios de formação desportiva.

\section{INTRODUCCIÓN}

La formación deportiva es un proceso que debe ocurrir a largo plazo (Côté y Hay, 2002), en el que influyen diferentes factores, principalmente en lo que se refiere a la manera en como los niños y los jóvenes se involucran con la práctica deportiva. Este proceso está relacionado, principalmente, con las vivencias y experiencias motoras, con la preparación diversificada y específica en un deporte (Coté, Lidor y Hackfort, 2009; Marques, Lima, Moraes, Nunomura y Simões, 2014), y con las influencias externas a que es sometido el deportista.

Con el fin de buscar la sistematización de una formación deportiva eficiente, Côté, Turnnidge y Vierimaa (2016) destacan los tres elementos dinámicos de la formación deportiva, los cuales están relacionados con participación personal en actividades, la calidad de las relaciones interpersonales y los contextos apropiados de práctica deportiva. Estos elementos tienen en cuenta las actividades físicas y deportivas practicadas desde la fase inicial del desarrollo motor hasta el rendimiento deportivo, así como las interacciones con las personas y los contextos de práctica deportiva. La diversificación deportiva precoz, las altas cantidades de juego deliberado, la centralización en los niños y el estar rodeados de pares involucrados con el deporte, parecen ser características esenciales del ambiente para alentar a los jóvenes a la posterior inversión en la práctica de actividades estructuradas (Côté y Vierimaa, 2014).

En este sentido, diversos autores han destacado la importancia de la práctica de un elevado número de actividades variadas antes de especializarse en un solo deporte (Collet, Nascimento, Folle y Ibáñez, 2017; Côté et al., 2016; Ericsson, Krampe y TeschRomer, 1993), de la obtención de incentivo y apoyo de las personas más cercanas (Côté, 1999; FraserThomas, Strachan y Jeffery-Tosoni, 2013; Keegan, Harwood, Spray y Lavallee, 2009; Holt y Neely, 2011), así como de la participación en contextos adecuados de práctica (Balish y Côté, 2013; Blázquez-Manzano, 2011; Macdonald y Baker, 2013; Pazo, Saénz-López y Fradua, 2012). Estas investigaciones han demostrado la importancia de estos elementos dinámicos por medio de estudios teóricos y empíricos con jugadores y practicantes de diferentes modalidades y en diferentes países, incluso relacionados al voleibol (Coutinho, 2014; Marques et al., 2014).

El voleibol se ha destacado por su gran alcance en el número de practicantes y espectadores, principalmente en Brasil, en virtud de la relevancia de las selecciones nacionales por medio de la consecución de éxitos en varios campeonatos en diferentes categorías y género. Los datos de la Federación Internacional de Voleibol (FIVB) de 2017 colocan a Brasil en el primer lugar en el ranking mundial en la categoría sénior masculina y cuarto lugar en la categoría sénior femenina. Además, las categorías de base también se destacan en el escenario internacional, pues todas las selecciones están entre las diez mejores en el ranking mundial. Con el fin de mantener el nivel de excelencia de las selecciones y el alcance de los practicantes, es necesario comprender cada vez más los aspectos determinantes para una formación deportiva de calidad. 
Collet, C., Nascimento, J. V., Folle, A., Ibáñez, S. J.

El análisis del proceso de formación deportiva de jugadores de élite puede revelar aspectos que favorezcan la formación de nuevos jugadores para el alto rendimiento, así como de practicantes y personas implicadas con el deporte en sus diferentes manifestaciones. Sin embargo, la recogida de información de esta naturaleza depende de la utilización de instrumentos que posibiliten la obtención de datos confiables y adecuados a lo que se pretende comprobar. En este contexto, los instrumentos utilizados para tales fines deben seguir un proceso que va desde la construcción de sus dimensiones y componentes hasta la comprobación de su validez y fiabilidad (Drost, 2011; HernándezSampieri, Fernández-Collado y Baptista-Lucio, 2014; Polit y Beck, 2003), antes de que sean empleados en la población.

Los investigadores diseñan instrumentos que les permitan obtener evidencias sobre su objeto de estudio. Una vez diseñados y validados, estos instrumentos pueden ser aplicados por otros investigadores en contextos semejantes. En la literatura deportiva, se han realizado muchos estudios con métodos de validez para cuestionarios sobre la formación inicial en Educación Física (CastejónOliva, Santo-Pastor y Palacios-Picos, 2015), la autoimagen corporal (Lucena-Santos, Carvalho, Silva Oliveira y Pinto-Gouveia, 2017), pérdida de peso en deportes de combate (Martínez-Abellán et al., 2016), y sobre la actividad física (Manchola-González, Bagur-Calafat y Girabent-Farrés, 2017; Mendo, 2001). Se han validado también escalas para medir el estrés (Lima et al., 2016) y clima motivacional en actividades físico-deportivas (Moreno, López, Martínez-Galindo, Alonso y González-Cutre, 2007), bien como sistemas de observación en voleibol (Collet, Nascimento, Ramos y Stefanello, 2011), baloncesto (Folle et al., 2014) y fútbol para ciegos (Gamonales, León, Muñoz, González y Ibáñez, 2018). Se pueden encontrar tambien validaciones de programas de intervención educativa (González, Ibáñez, Feu y Galatti, 2017), instrumentos obsevacionales para el análisis de juego en rugby (Villarejo, Ortega, Gómez y Palao, 2014), el rendimiento en fútbol (García-López, GonzálezVíllora, Gutiérrez y Serra, 2013), el conocimiento táctico ofensivo en fútbol (Serra-Olivares y GarcíaLópez, 2016), o la intervención de los árbitros en baloncesto (García-Santos y Ibáñez, 2016). También se encuentran herramientas que indagan sobre las preferencias y satisfacción de los deportistas en sus actuaciones especiales de juego (Ortega, Jiménez, Palao y Sáinz de Barranda, 2008), o cuestionarios sobre los factores socio-emocionales que influyen en la progresión de los jóvenes futbolista (GómezCarmona, Cervera y Benito, 2014).

Son escasas las herramientas que permitan analizar los procesos de formación deportiva. García-Martín, Antúnez e Ibáñez (2016) diseñan y validan una batería de instrumentos de medición (ATPEP), compuesta por dos entrevistas cualitativas y una escala, para identificar y analizar el proceso de formación que han seguido los deportistas para llegar a ser expertos, aplicable a diferentes deportes colectivos. Por su parte Robles-Rodríguez, RoblesRodríguez, Fuentes-Guerra y Abad-Robles (2016) validan una entrevista cualitativa para conocer cómo es el proceso de formación de los judokas españoles. El guión de entrevistas sugerido por Côté, Ericsson y Law (2005) y utilizado por Coutinho (2014), se focaliza en diferentes factores que pueden afectar al rendimiento, como logro de éxitos en el deporte específico, participación en otras actividades deportivas, lesiones, crecimiento físico y calidad de los recursos en cada año de entrenamiento. Estos instrumentos, aún siendo específicos para indagar en los procesos de formación deportiva, no permiten conocer los elementos dinámicos de la formación deportiva, vinculados con participación en diversas actividades, la calidad de las relaciones interpersonales y los contextos de práctica deportiva.

Los instrumentos utilizados para la comprobación de los diferentes aspectos de la formación deportiva consisten, en su mayoría, de entrevistas con análisis cualitativos (Côté et al., 2005; Coutinho, 2014), cuestionarios que buscan informaciones específicas o demasiado amplias, que no atienden el análisis de la formación deportiva a partir del abordaje de sus elementos dinámicos, tal y como definen Côté et al. (2016). Ante la ausencia de un instrumento para recogida de informaciones que abarque los elementos dinámicos de la formación deportiva en sus diferentes etapas, el objetivo general del presente estudio fue construir y comprobar la validez de contenido y la fiabilidad (estabilidad y consistencia interna) del Instrumento de Análisis de la Formación Deportiva en Voleibol (IAFDV), elaborado a partir de los 


\section{Construcción y validación de un instrumento para el análisis de la formación deportiva en voleibol}

elementos dinámicos propuestos por Côté et al., (2016), y de las etapas de formación deportiva destacadas por Côté (1999). Para ello, los objetivos específicos fueron: i) establecer los componentes y las cuestiones del instrumento; ii) validar el contenido a partir de la evaluación de jueces expertos; iii) analizar su fiabilidad a partir del análisis de la estabilidad y consistencia interna del instrumento.

\section{MATERIAL Y MÉTODOS}

\section{Diseño}

El presente estudio se clasifica como estudio instrumental (Ato, López y Benavente, 2013; Montero y León, 2007), que tiene como objetivo el desarrollo de pruebas y aparatos, incluyendo el diseño o la adaptación y el estudio de sus propiedades psicométricas. El proceso de validación del presente instrumento de medida siguió las recomendaciones de la literatura (Cassepp-Borges, Balbinotti y Teodoro, 2009; Drost, 2011; HernándezSampieri et al., 2014), las cuales indican que los instrumentos de medición deben ser válidos y fiables, por medio de diferentes maneras de obtener los coeficientes de cada aspecto. En el caso de los componentes y de las preguntas del instrumento, éste pasó por el proceso de validación de contenido (Cassepp-Borges et al., 2009), y por el proceso de comprobación de la fiabilidad por medio de la estabilidad y de la consistencia interna (CarreteroDios y Peres, 2005; Drost, 2011).

\section{Participantes}

La construcción inicial del instrumento contó con la participación de investigadores con experiencia en el voleibol y en los temas relacionados a la Pedagogía del Deporte y la formación deportiva. El procedimiento de validación se realizó a partir del método de juicio de expertos (Cassepp-Borges et al., 2009; Escobar-Pérez y Cuervo-Martínez, 2008; Escurra, 1988), con alto conocimiento en la materia, y que debían cumplir ciertos criterios de selección (Skjong y Wentworth, 2001). La población seleccionada para este estudio fueron 15 jueces expertos que cumplían al menos a cinco de los seis criterios establecidos para la selección como evaluadores: i) ser doctor en Educación Física o Deporte; ii) ser profesor de enseñanza superior en universidades brasileñas; iii) ser investigador con publicaciones en las áreas de la Pedagogía del Deporte y de la formación deportiva; iv) haber actuado como entrenador de categorías de base o como profesor de la Educación Física Escolar, visando la formación deportiva de jugadores; v) enseñar o haber enseñado materias relacionadas con la Pedagogía del Deporte, Educación Física Escolar o modalidades deportivas específicas en la Enseñanza Superior; vi) tener publicaciones relacionadas con la construcción y la validación de instrumentos de evaluación. Finalmente, la muestra para la validación de contenido estuvo compuesta por 12 jueces expertos que contestaron con la validación, de forma satisfactoria y con la cantidad de información recomendada por la literatura (Lynn, 1986).

La Tabla 1 presenta los criterios de inclusión que cumplieron cada uno de los evaluadores expertos que componen la muestra. La información referente a los evaluadores fue obtenida a través de la consulta de los currícula disponibles en la Plataforma Lattes / $\mathrm{CNPq} /$ Brasil, por tratarse de un sistema de dominio público.

Tabla 1. Cumplimiento de los criterios de selección de los evaluadores del instrumento.

\begin{tabular}{|c|c|c|c|c|c|c|c|c|c|c|c|c|c|}
\hline \multicolumn{14}{|c|}{ Jueces evaluadores } \\
\hline \multirow{7}{*}{ Criterios } & & 1 & 2 & 3 & 4 & 5 & 6 & 7 & 8 & 9 & 10 & 11 & 12 \\
\hline & $I$ & $\sqrt{ }$ & $\sqrt{ }$ & $\sqrt{ }$ & $\checkmark$ & $\checkmark$ & $\sqrt{ }$ & $\sqrt{ }$ & $\sqrt{ }$ & $\checkmark$ & $\sqrt{ }$ & $\checkmark$ & $\checkmark$ \\
\hline & II & $\checkmark$ & - & $\checkmark$ & $\checkmark$ & $\checkmark$ & $\sqrt{ }$ & $\checkmark$ & $\sqrt{ }$ & $\checkmark$ & $\checkmark$ & $\checkmark$ & $\checkmark$ \\
\hline & $I I I$ & $\checkmark$ & $\sqrt{ }$ & $\sqrt{ }$ & $\sqrt{ }$ & $\checkmark$ & $\checkmark$ & $\sqrt{ }$ & $\sqrt{ }$ & $\checkmark$ & $\sqrt{ }$ & $\checkmark$ & $\checkmark$ \\
\hline & IV & - & $\checkmark$ & $\checkmark$ & $\checkmark$ & $\checkmark$ & $\checkmark$ & $\checkmark$ & $\checkmark$ & $\checkmark$ & $\checkmark$ & $\checkmark$ & - \\
\hline & $V$ & $\sqrt{ }$ & $\sqrt{ }$ & $\sqrt{ }$ & $\sqrt{ }$ & $\checkmark$ & $\sqrt{ }$ & $\sqrt{ }$ & $\sqrt{ }$ & - & $\sqrt{ }$ & $\sqrt{ }$ & $\checkmark$ \\
\hline & $V I$ & $\sqrt{ }$ & $\sqrt{ }$ & - & $\checkmark$ & $\checkmark$ & $\sqrt{ }$ & - & - & $\checkmark$ & - & $\sqrt{ }$ & $\checkmark$ \\
\hline
\end{tabular}

Para la comprobación preliminar de la fiabilidad a través de la estabilidad y de la consistencia interna del IAFDV participaron 15 jugadores de dos equipos de formación deportiva de un club de Brasil, participantes en campeonatos estatales de voleibol, con una media de edad de 16,74+1,03 años. La elección de los participantes de esta etapa fue intencional (Hernández-Sampieri et al., 2014), teniendo en cuenta la mayor accesibilidad y la posibilidad de aplicación del instrumento en dos ocasiones, contando con la aceptación y el consentimiento de los dirigentes del club, del entrenador de los jugadores y sus responsables o 
Collet, C., Nascimento, J. V., Folle, A., Ibáñez, S. J.

tutores legales (en el caso de los jugadores menores de 18 años).

\section{Variables}

Las variables del estudio fueron la validez de contenido y la fiabilidad del instrumento. La validez de contenido fue determinada a través de tres dimensiones (Cassepp-Borges et al., 2009): claridad del lenguaje/redacción (se refiere a los términos y el lenguaje utilizado para determinar cada cuestión del instrumento); pertinencia práctica (si tiene importancia para lo que el instrumento se propone a evaluar), y relevancia teórica (referente al nivel de asociación entre la cuestión y la teoría). Los evaluadores del instrumento marcaron, en una escala de 1 (muy adecuada) a 5 (inadecuada), el grado de validez de cada cuestión en las tres dimensiones. Además de la cuantificación del nivel de adecuación de cada pregunta, los evaluadores pudieron expresar sus opiniones o sugerencias con el fin de mejorar los ítems del instrumento. La fiabilidad del instrumento fue determinada a través de la comprobación de la estabilidad (método de test-retest), y de la consistencia interna del instrumento (Carretero-Dios y Peres, 2005; Drost, 2011).

\section{Instrumentos}

El IAFDV se constituyó en dos partes: la primera parte sobre los datos sociodemográficos de los jugadores, tales como el sexo, la fecha y el lugar de nacimiento, la escolaridad, así como información sobre el lugar donde pasó la infancia, preadolescencia y la adolescencia, sobre el inicio de la participación en el voleibol sistematizado, las funciones del voleibol en que actúa y actuó y el nivel de las competiciones internacionales en las que participó. La segunda parte está subdividida en tres etapas, separadas a partir de los períodos de la formación deportiva (Côté, 1999): Años de Diversificación (infancia - hasta 12 años); Años de Especialización (preadolescencia - de 13 a 15 años) y Años de Inversión (adolescencia - de 16 a 18 años) para facilitar el proceso recordatorio de los jugadores. En cada grupo de edad, las cuestiones versaban sobre los elementos dinámicos del desarrollo deportivo, a partir del modelo teórico de Côté et al. (2016). Estos elementos abarcan la participación personal en actividades, la calidad de las relaciones interpersonales y los escenarios apropiados para el desarrollo deportivo. El cuestionario se construyó a partir de una matriz analítica (Tabla 2) que presenta los objetivos, las dimensiones y los indicadores para las cuestiones.

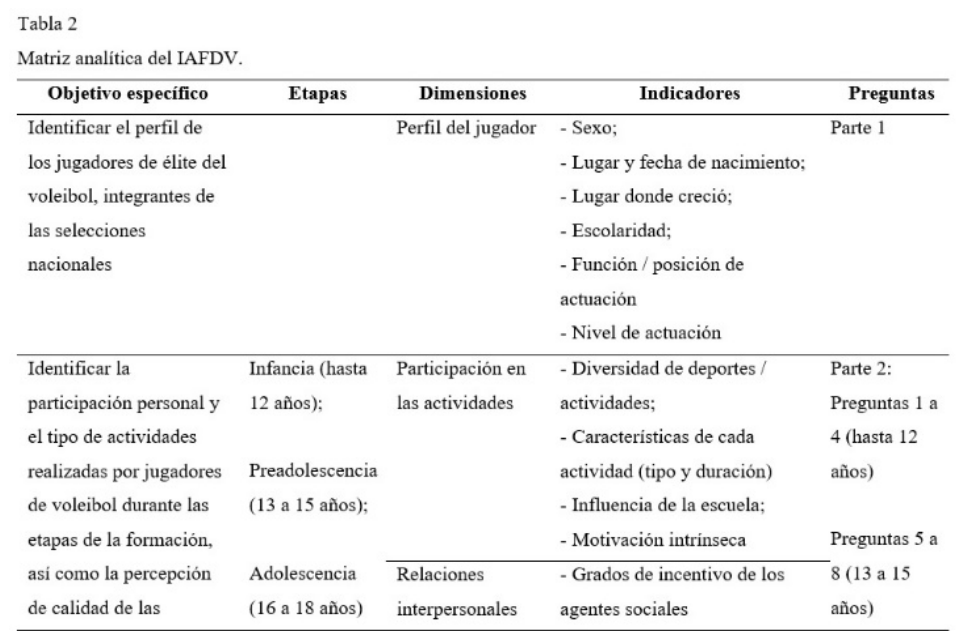

El IAFDV tiene preguntas abiertas y cerradas en las que se pueden obtener diferentes informaciones. Así, las 12 preguntas evaluadas conjuntamente en el proceso de validación de contenido permitieron obtener 78 datos específicos de los jugadores, transformados en variables para el posterior análisis cuantitativo. Estas variables se utilizaron para comprobar la fiabilidad a partir de la estabilidad y consistencia interna del instrumento.

\section{Procedimiento}

Después de la construcción del instrumento, se inició el proceso de comprobación de su validez (validez de contenido) y fiabilidad (estabilidad y consistencia interna). Los sujetos para comprobar la validez de contenido deberían tener experiencia en el tema, conocimiento y cualificación y que podrían proporcionar informaciones, evidencias, juicios y evaluaciones (Escobar-Pérez y Cuervo-Martínez, 2008), aspectos contemplados a partir de los criterios de selección. Los jueces seleccionados recibieron los cuestionarios, vía correo electrónico, conjuntamente con una carta de invitación para participar, conteniendo las explicaciones y los objetivos de la investigación. En el caso de acuerdo, deberían asignar un valor en la escala de 1 a 5 (evaluación cuantitativa) para las dimensiones de la claridad del lenguaje, la pertinencia práctica y la relevancia 


\section{Construcción y validación de un instrumento para el análisis de la formación deportiva en voleibol}

teórica de su contenido (Cassepp-Borges et al., 2009), así como proporcionar sugerencias si así lo deseaban (evaluación cualitativa). Las respuestas con las evaluaciones fueron recibidas dentro del plazo de un mes de 12 de los 15 expertos, siendo compiladas en documento único las evaluaciones y las sugerencias de perfeccionamiento de cada pregunta, para facilitar la organización de las sugerencias de alteración en cada cuestión.

Después del proceso de validación de contenido y las modificaciones a partir de las sugerencias de los jueces expertos, la versión final del instrumento fue contestada por dos equipos de jugadores de categorías de formación que acordaron participar de la investigación, en dos ocasiones (método test-retest) con un intervalo de una semana entre las aplicaciones para comprobar la fiabilidad del instrumento.

\section{Análisis estadístico}

El Índice de Validez de Contenido (IVC) fue determinado por el V Aiken (Aiken, 1985) a partir de la ecuación modificada por Penfield y Giacobbi (2004) y con la ayuda del Programa Visual Basic 6 desarrollado por Merino y Livia (2009). El IVC tiene una magnitud de 0 a 1 , siendo el 1 la mayor correlación entre dos variables. Los valores mínimos aceptables son fijos y determinados a partir del número de jueces expertos o del número de cuestiones del instrumento. En el presente estudio el IVC establecido para el número de evaluadores fue de, $69(\mathrm{p}=, 041)$ y atendiendo al número de ítems fue de, $75(\mathrm{p}=, 006)$ (Aiken, 1985).

La ecuación algebraicamente modificada de Penfield y Giacobbi (2004) para el cálculo del índice (V) considera la media de las calificaciones de los jueces expertos $(\dot{X})$, la calificación más baja (1) y el intervalo de posibles respuestas de la escala $(\mathrm{k}=$ máximo - mínimo), en el caso del presente estudio, $\mathrm{K}$ $=5-1=4$.

$$
V=\frac{\bar{X}-l}{k}
$$

El cálculo del IVC se realizó con la ayuda del programa informático Visual Basic 6 que proporciona los valores de $\mathrm{V}$ y permite la obtención de Intervalo de Confianza (IC) al 90\%, 95\% y 99\%.
En el presente estudio, se optó por la utilización del IC95\%, teniendo en cuenta la recomendación para los estudios en el área de las Ciencias Sociales (Merino y Livia, 2009).

Para analizar la fiabilidad del instrumento se realizaron las pruebas de estabilidad y consistencia interna. Para analizar la estabilidad de la respuesta se empleó un test-retest (Drost, 2011; HernándezSampieri et al., 2014). Los datos del test-retest fueron analizados a partir de la correlación de Spearman para variables no paramétricas, lo que permitió comprobar la estabilidad temporal del instrumento (Hernández-Sampieri et al., 2014). La consistencia interna define cuánto un conjunto de elementos mide un comportamiento o característica particular en la prueba, con estimaciones de fiabilidad basadas en las interrelaciones medias entre todos los ítems individuales (Drost, 2011), y que puede obtenerse a partir del coeficiente Alpha de Cronbach (Cronbach, 1951). En ambos cálculos se utilizó el paquete estadístico SPSS versión 23, adoptando el nivel de significancia del $5 \%(\mathrm{p}<, 05)$.

\section{RESULTADOS}

Los resultados del proceso de validación del IAFDV se describen a continuación, los cuales muestran los IVC de cada pregunta para determinar la validez, así como los coeficientes de estabilidad y de consistencia interna para la definición de la fiabilidad del instrumento. La Tabla 3 muestra los datos de validación de contenido (V de Aiken e IC 95\%) en lo que se refiere a la claridad del lenguaje, la pertinencia práctica y la relevancia teórica de cada una de las preguntas. 
Collet, C., Nascimento, J. V., Folle, A., Ibáñez, S. J.

Tabla 3

Indices de Validez de Contenido $V$ de Aiken e Intervalos de Confianza del $95 \%$.

\begin{tabular}{|c|c|c|c|c|c|c|c|c|c|c|}
\hline & \multicolumn{3}{|c|}{ Claridad de lenguaje } & \multicolumn{3}{|c|}{ Pertinencia práctica } & \multicolumn{4}{|c|}{ Relevancia teórica } \\
\hline & \multirow[b]{2}{*}{$V$} & \multicolumn{2}{|c|}{ IC95\% } & \multicolumn{3}{|c|}{ IC95\% } & \multirow[b]{2}{*}{ V } & \multicolumn{2}{|c|}{ IC95\% } & \\
\hline & & Min. & Máx. & V & Min. & $M a x$. & & Min. & Máx. & \\
\hline Parte 1 & ,94 & 83 &, 80 & ,92 &, 80 & ,97 & ,94 & 83 & ,98 & ,93 \\
\hline Pregunta 1 & ,94 & ,83 & ,98 & ,90 &, 78 & ,95 &, 85 & ,73 & ,93 & ,90 \\
\hline Pregunta 2 & ,96 & ,86 & ,99 &, 90 & ,78 & ,95 & 88 &, 75 & ,94 & ,91 \\
\hline Pregunta 3 & ,92 &, 80 & ,97 & ,90 &, 78 & ,95 & 88 &, 75 & ,94 & ,90 \\
\hline Pregunta 4 & 90 & ,78 & ,95 & ,90 &, 78 & ,95 & ,90 & ,78 & ,95 & ,90 \\
\hline Pregunta 5 & 94 & 83 & ,98 & ,92 &, 80 & ,97 & ,92 & 80 & ,97 & 93 \\
\hline Pregunta 6 & 96 & 86 & ,99 & ,90 &, 78 & ,95 & 88 & ,75 & ,94 &, 91 \\
\hline Pregunta 7 & ,92 & ,80 & ,97 &, 92 &, 80 & ,97 & ,90 &, 78 & ,95 & ,91 \\
\hline Pregunta 8 & 90 & ,78 & ,95 & ,90 &, 78 & ,95 & ,90 & ,78 & ,95 &, 90 \\
\hline Pregunta 9 & ,94 & 83 & ,98 &, 92 &, 80 & ,97 & ,92 & 80 & ,97 & ,93 \\
\hline Pregunta 10 & 96 & 86 & ,99 &, 85 &, 73 & 93 & 83 & ,70 & ,91 &, 88 \\
\hline Pregunta 11 & ,92 & ,80 & ,97 &, 92 &, 80 & ,97 & ,90 & ,78 & ,95 & ,91 \\
\hline Pregunta 12 & ,90 & ,78 & 95 & ,90 &, 78 & ,95 & ,90 & ,78 & ,95 & ,90 \\
\hline Total & ,93 &, 82 & ,96 & 90 &, 78 & ,96 &, 89 &, 77 &, 95 & ,91 \\
\hline
\end{tabular}

Todos los IVC determinados por el $\mathrm{V}$ de Aiken fueron mayores que los valores de referencia, tanto en lo que se refiere al número total de evaluadores $(\mathrm{V}$ $>, 69 ; \mathrm{p}=, 041)$, como en el número de ítems (V $>, 75 ; \mathrm{p}=, 006)$, indicando un alto índice de validez del instrumento. El IVC general del cuestionario fue de, 91 .

Las evaluaciones cualitativas procedentes de las sugerencias de los jueces expertos se analizaron conjuntamente para cada ítem/pregunta y para todo el instrumento, que se añadieron o modificaron en el cuestionario final. La principal sugerencia, que fue acatada, fue la de incluir una pregunta sobre las actividades realizadas durante las clases de Educación Física en la escuela, para que los jugadores pudieran indicar cuánto influyen esas actividades en su interés y desarrollo deportivo.

En la Tabla 4 se presentan los resultados correspondientes a la fiabilidad del IAFDV, a partir de la estabilidad y la consistencia interna de cada ítem. Se observa que 77 de los 78 ítems del instrumento tienen estabilidad significativa ( $p$ $<, 005)$, siendo que 67 presentaron correlación superior a ,70 y 61 por encima de ,80. Se observó también alta consistencia interna, pues 77 de los 78 ítems tienen valor superior a ,70 $(\alpha>, 70)$. Además, 11 de los ítems presentaron valores entre ,50 y ,70, 21 presentaron valores entre ,70 y ,90 y 46 de los ítems obtuvieran valores de correlación superior a ,90.
Tabla 4

Datos referentes a la eatabilidad ya la consistencia interna de los items de cada cuestión del instrumento. Años de diversificación Años de especialización Años de Inversión $\begin{array}{lll}\text { (hasta los } 12 \text { años) } & \text { (13 } \mathrm{a} 15 \text { años) } & (16 \mathrm{a} 18 \text { años) }\end{array}$

\begin{tabular}{|c|c|c|c|c|c|c|c|c|c|c|c|c|}
\hline & \multicolumn{4}{|c|}{ Bxegunta 1} & \multicolumn{4}{|c|}{ Pregunta 5} & \multicolumn{4}{|c|}{ Pregunta, 9} \\
\hline & tho & & $p$ & $\alpha$ & rho & & $p$ & $\alpha$ & the & & $p$ & $\alpha$ \\
\hline A &, 783 & * & ,002 & ,884 & .584 & $\bullet$ &, 022 &, 793 & 0,624 & $\bullet$ &, 04 &, 789 \\
\hline B &, 895 & $*$ & $<, 001$ & ,948 & 1 & $*$ & $<, 001$ & 1 & 0,856 & $\omega$ &, 001 & 940 \\
\hline c & 1 & $*$ & $<, 001$ & 1 & 1 & $*$ & $<, 001$ &, 975 & 1 & $*$ & $<, 001$ & 1 \\
\hline D &, 732 & - &, 002 & 960 & 1 & 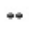 & $<, 001$ & 1 & 1 & $\bullet$ & $<, 001$ & 1 \\
\hline \multirow[t]{3}{*}{ E } &, 752 & $*$ &, 001 &, 787 & 1 & * & $<, 001$ & 960 & 1 & 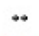 & $<, 001$ & 1 \\
\hline & \multicolumn{4}{|c|}{ Pregunta 2} & \multicolumn{4}{|c|}{ Pregunta 6} & \multicolumn{4}{|c|}{ Pregunta 10} \\
\hline & the & & $p$ & $a$ & rho & & $p$ & $\alpha$ & tho & & $p$ & $\alpha$ \\
\hline A & 1 & 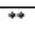 & $<, 001$ & 1 & ,916 & $\cdots$ & $<, 001$ & 968 & 1 & $*$ & $<, 001$ & 1 \\
\hline B & ,999 & $*$ & $<, 001$ & 998 & 1 & • & $<, 001$ &, 988 & ,908 & $*$ & $<, 001$ & ,992 \\
\hline c & 1 & $*$ & $<, 001$ & 1 & .967 & • & $<, 001$ & .905 & 1 & $*$ & $<, 001$ & 1 \\
\hline D & ,925 & $*$ & $<, 001$ & 959, &, 737 & - &, 002 &, 926 & 867 & 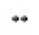 & ,001 & 949 \\
\hline E &, 832 & $*$ & $<, 001$ & ,962 &, 690 & • &, 004 & ,934 & 683 & - &, 020 & ,718 \\
\hline$F$ & 949 & $*$ & $<, 001$ & 992 &, 545 & - & .036 &, 717 & 801 & . &, 003 & 897 \\
\hline
\end{tabular}

\begin{tabular}{|c|c|c|c|c|c|c|c|c|c|c|c|c|}
\hline & tho & & $p$ & $\alpha$ & rho & & $p$ & $\alpha$ & tho & & $p$ & $\alpha$ \\
\hline $\mathbf{A}$ & ,990 & $*$ & $<, 001$ & 981 & 885 & $\cdots$ & $<, 001$ & ,936 & ,903 & $*$ & $<, 001$ & ,963 \\
\hline B & 1 & $*$ & $<, 001$ & 1 & 824 & $\cdots$ & $<, 001$ & ,883 &, 627 & - & ,039 & ,667 \\
\hline c & ,988 & $*$ & $<, 001$ & ,985 & ,699 & 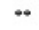 & ,004 &, 873 & ,921 & $*$ & $<, 001$ & ,987 \\
\hline D &, 902 & $*$ & $<, 001$ & ,976 & 940 & 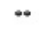 & $<, 001$ & ,981 & 997 & * & $<, 001$ & ,985 \\
\hline E & ,912 & $*$ & $<, 001$ & 966 & ,935 & 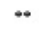 & $<, 001$ &, 977 &, 997 & $*$ & $<, 001$ & ,994 \\
\hline F & ,968 & $*$ & $<, 001$ & ,982 & ,960 & 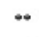 & $<, 001$ & ,981 & ,935 & 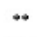 & $<, 001$ & 918 \\
\hline
\end{tabular}

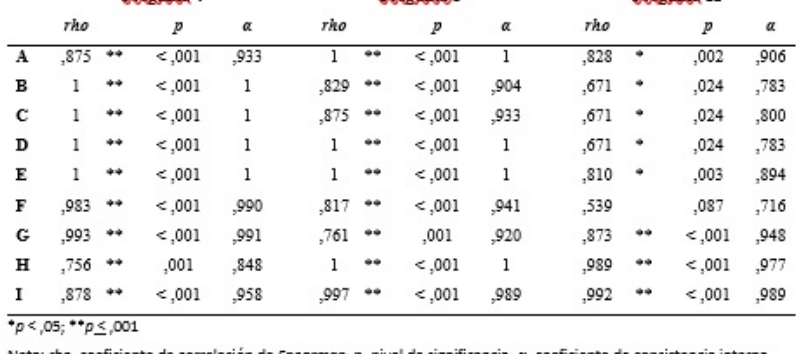

Nota: rha- coeficiente de correlación de Spearman; p-nivel de significancia; $\alpha$ - coeficiente de consistencia interna.

\section{DISCUSIÓN}

El objetivo del estudio fue construir y validar un cuestionario (IAFDV) para obtener información sobre la formación deportiva de los jugadores brasileños de voleibol, el cual presentó altos índices de validez y fiabilidad. En la construcción, se llevaron a cabo la consulta en literatura específica acerca de los elementos dinámicos de la formación deportiva (Côté et al., 2016) y se utilizaron como base las entrevistas que abordaban algunos aspectos específicos de la formación deportiva (Côté et al., 2005; Coutinho, 2014), relacionados con la participación en actividades físicas y deportivas, las relaciones interpersonales y las influencias de los agentes sociales más cercanos, así como la inserción en contextos adecuados de práctica. La división del cuestionario en dos partes facilita la recogida de informaciones sobre los datos sociodemográficos de los jugadores y sobre características de las etapas de formación deportiva (Côté, 1999). Informaciones sobre tales elementos favorecen el desarrollo positivo 


\section{Construcción y validación de un instrumento para el análisis de la formación deportiva en voleibol}

de jóvenes insertos en el contexto deportivo (Holt y Neely, 2011).

Para que el cuestionario sea científicamente utilizable, se realizaron los procedimientos para comprobar la validez a través de la validación de contenido y la fiabilidad a través de la estabilidad y consistencia interna, como sugiere la literatura específica del área (Cassepp-Borges et al., 2009; Drost, 2011 y Hernández-Sampieri et al., 2014). Métodos similares se utilizaron en un gran número de estudios para la construcción y validación de instrumentos de investigación, como cuestionarios (Castejón-Oliva et al., 2015; Lucena-Santos et al., 2017; Manchola-González et al., 2017; MartínezAbellán et al., 2016), entrevistas (García-Martín et al., 2016; Robles-Rodríguez et al., 2016; Bohórquez Gómez-Millán y Checa Esquiva 2017), escalas (Lima et al., 2016) y sistemas de observación (Collet et al., 2011; Folle et al., 2014; Gamonales et al., 2018 y García-Santos y Ibáñez, 2016; Villarejo et al., 2014).

El proceso de validación de instrumentos, por medio de la evaluación de jueces expertos realizado en el presente estudio también ha sido utilizado en estudios relacionados con la formación deportiva (Cronin y Allen, 2017; García-Martín et al., 2016; RoblesRodríguez et al., 2016 y Weiss, Volter y Kipp, 2014) y con la psicología del deporte (Bohórquez GómezMillán y Checa Esquiva 2017). Este método describe el grado en que un instrumento realmente mide la variable de interés, de acuerdo con expertos en el tema (Escobar-Pérez y Cuervo-Martínez, 2008 y Hernández-Sampieri et al., 2014). Así, se ha seguido un proceso meticuloso y validado para determinar la validez de contenido y el nivel de adecuación de las preguntas proporcionado por los jueces expertos, y que afecta la calidad del instrumento.

Las indicaciones sobre la cantidad de jueces expertos necesarios para validar un instrumento varían dependiendo de las características del instrumento y de los objetivos que se pretenden alcanzar (EscobarPérez y Cuervo-Martínez, 2008). El presente estudio contó con la colaboración de 12 jueces expertos, lo que se considera satisfactorio para estudios de esta naturaleza, pues la literatura recomienda emplear un número cercano a 10 (Escurra, 1988, Hyrkäs, Appelqvist-Schmidlechner y Oksa, 2003), y similar al número de jueces utilizados en los estudios de Collet et al. (2011); Folle et al. (2014); García-Martín et al. (2016); Robles-Rodríguez et al. (2016) y Villarejo et al. (2014). La selección de los jueces expertos se realizó sobre la base de criterios relacionados con la cualificación, experiencia, publicaciones, accesibilidad, imparcialidad y reconocido conocimiento (Escobar-Pérez y CuervoMartínez, 2008 y Skjong y Wentworth, 2001). Los jueces seleccionados atendieron al menos a 5 de los 6 criterios de selección, corroborando con la recomendación de que los evaluadores deben cumplir al menos el $80 \%$ de los criterios establecidos (Voutilainen y Liukkonen, 1995). De ese modo, la validez del contenido del instrumento es certificada por un número adecuado de jueces de alta calidad en Ciencias del Deporte.

Los procedimientos utilizados para comprobar la validez del IAFDV a partir del grado de adecuación en las dimensiones de claridad de lenguaje, pertinencia práctica y relevancia teórica también se utilizaron en los estudios de Collet et al. (2011), Folle et al. (2014), Lima et al. (2016) y Lucena-Santos et al. (2017). Los datos procedentes de la evaluación cuantitativa de cada cuestión se agruparon y se calcularon el índice de validez de contenido $\mathrm{V}$ de Aiken (Aiken, 1985), que proporciona una estimación de la calidad del contenido del instrumento. De acuerdo con Escurra (1988), ese índice es el más adecuado para determinar la validez de contenido, pues permite obtener valores estadísticos según el tamaño de la muestra. Además, es ampliamente utilizado en estudios sobre validación de instrumentos (v.g., García-Martín et al., 2016, González et al., 2017, Robles-Rodríguez et al., 2016, Villarejo et al., 2014). Herramientas informáticas se utilizan para el cálculo de diversos coeficientes para facilitar su ejecución y buscando descartar las posibilidades de errores, que pueden ocurrir cuando se calculan manualmente.

Los coeficientes $\mathrm{V}$ de Aiken de todas las cuestiones del instrumento cumplen valores por encima del valor de referencia exacto para el nivel crítico aceptable. Estos índices indican un alto nivel de aceptación en el contenido del instrumento, adecuado para medir el proceso de formación deportiva de los jugadores de voleibol. La validez de contenido es muy alta, mostrando que los ítems diseñados son útiles para identificar cómo influyen los tres elementos dinámicos de la formación deportiva en los jugadores 
Collet, C., Nascimento, J. V., Folle, A., Ibáñez, S. J.

de voleibol. Hasta la fecha, el modelo teórico propuesto por Côté et al. (2016), no disponía de una herramienta cuantitativa para su comprobación práctica, lo que permitirá una gran difusión, al economizar los tiempos que los investigadores necesitan para obtener esta información, frente a las técnicas cualitativas. Los valores de $\mathrm{V}$ de Aiken en las dimensiones (claridad de lenguaje, pertinencia práctica y relevancia teórica) de la validación de cada pregunta variaron desde ,83 hasta ,96, indicando que los jueces evaluadores consideraron esta herramienta adecuada para medir el proceso de formación deportiva de los jugadores de voleibol. Estos valores fueron similares a los encontrados en los estudios de García-Martín et al. (2016), de Villarejo et al. (2014), de Blázquez-Manzano (2011) y de Martínez-Abellán et al. (2016), los cuales encontraron valores $\mathrm{V}$ de Aiken por encima de ,79, ,85, ,88, y ,81, respectivamente. Los altos valores obtenidos ponen de manifiesto un correcto diseño del instrumento, siendo adecuados para analizar el constructo del modelo de formación deportiva propuesto por Coté et al. (2016), tanto en sus tres dimensiones, como en los diferentes períodos formativos.

Una vez obtenidos los valores cuantitativos de las evaluaciones de los jueces, se tuvieron en cuenta las evaluaciones cualitativas, a partir de los comentarios y sugerencias proporcionados por los jueces evaluadores para cada cuestión y para el instrumento en general. La mayoría de las aportaciones se focalizaron hacia clarificaciones conceptuales y mejora de expresiones gramaticales. Todas las sugerencias se acataron para mejorar la presentación y la secuencia lógica de las cuestiones. La sugerencia más relevante conceptualmente fue agregar un ítem acerca de las actividades en la escuela en cada etapa de la formación deportiva, teniendo en cuenta que las clases de Educación Física se constituyen de momentos propicios para el desarrollo integral del ser humano a partir de su participación en actividades (Flintoff, Foster y Wystawnoha, 2011 y González et al., 2017). El resultado final del cuestionario ha mejorado con las aportaciones ofrecidas por los expertos cuando se ha añadido una pregunta sobre las actividades realizadas durante las clases de Educación Física de la escuela, pues es un importante sitio de práctica deportiva en el contexto brasileño.
En lo que se refiere al análisis de la fiabilidad del instrumento IAFDV, realizado por el método testretest, los datos de la correlación entre las dos aplicaciones indicaron altos niveles de estabilidad en todos los ítems del instrumento. La estabilidad corresponde a la aplicación repetida de una prueba al mismo grupo de encuestados después de un intervalo de tiempo (Drost, 2011; Prieto y Delgado, 2010). El período de intervalo entre una aplicación y otro puede ser un factor limitante para investigaciones de esta naturaleza, pero consiste en un método importante para la verificación de la estabilidad del instrumento, para que sea fiable (Drost, 2011). La claridad del instrumento se ha puesto de manifiesto en la aplicación experimental del mismo con deportistas de las edades a las que va dirigido. Sus cuestiones han sido entendidas y contestadas de forma semejante tras un período de latencia. El cuestionario no sólo posee validez de contenido, sino que también es estable y fiable, una vez que, tras su cumplimentación en distintos momentos, se obtienen las mismas respuestas de los participantes.

La interpretación del grado de correlación depende de la característica del test, a partir de las implicaciones de las tomas de decisión, pero cuando se trata de investigaciones sociales, es posible tener como aceptables los valores de estabilidad cercanos y superiores a ,70 (Prieto y Delgado, 2010), o por encima de ,80 (Polit y Beck, 2003). En el presente estudio, los valores de correlación estuvieron, en su mayoría, por encima de estos valores de referencia y fueron categorizados de acuerdo con los valores establecidos por Crewson (2014), o sea, 46 de los ítems obtuvieran correlación muy alta, entre ,9 y 1, mientras que 21 obtuvieron una correlación alta y sólo 11 obtuvieran correlación moderada. Estos valores son similares al estudio de validación de cuestionario de Manchola-González et al. (2017) y de validación de entrevista de Robles-Rodríguez et al. (2016). En fin, los niveles de correlación fueron adecuados y suficientes para certificar la estabilidad del cuestionario y que las preguntas están diseñadas de manera clara y específica para obtener la información que se persigue.

La consistencia interna también indica la fiabilidad de un instrumento de evaluación, que en el caso del presente estudio fue utilizado el Alpha de Cronbach, por ser uno de los más conocidos y utilizados en el 


\section{Construcción y validación de un instrumento para el análisis de la formación deportiva en voleibol}

área de las Ciencias Sociales y Comportamentales (Drost, 2011). La consistencia interna de un instrumento determina cuán bien un grupo de elementos mide un comportamiento o característica particular dentro del test y se establece a partir de la intercorrelación media entre los elementos (Drost, 2011). En el presente estudio, los altos índices de consistencia interna indicaron que el cuestionario presenta coherencia interna en el establecimiento de los ítems, pues presentaron valores cercanos a ,70 y muchos más de ,80. Otros estudios presentaron valores similares, con consistencia interna general de ,838 (Castejón-Oliva et al., 2015), ,83 (MancholaGonzález et al., 2017), ,82 (Latorre y Pantoja, 2013) y ,843 (Cordente, González-Víllora, Pastor-Vicedo y Contreras, 2018). Los valores cercanos a 0,70 son aceptables y adecuados, siendo que en niveles más exigentes se esperan valores superiores a ,80 (Polit y Beck, 2003). Estos resultados indican que el cuestionario es fiable no sólo por su estabilidad, sino también por la consistencia interna obtenida por las respuestas de los participantes, pues las preguntas miden adecuadamente las características e informaciones que se busca obtener.

\section{CONCLUSIONES}

De manera general, las evidencias encontradas en el estudio permiten afirmar que el IAFDV es un instrumento válido y confiable para obtener informaciones sobre la formación deportiva de los jugadores de voleibol en general y brasileños en particular. La construción del instrumiento con las contribuiciones de los expertos y la verificación de su validación posibilitan la obtención adecuada de informaciones sobre los aspectos que más influyen en la formación deportiva de jugadores de voleibol. Así, se puede decir que ese es un instrumento adecuado para conocer aspectos relacionados a los elementos dinámicos de la formación deportiva de jugadores y jugadores de voleibol, teniendo una gran aplicabilidad.

El IAFDV, así como otros instrumentos que buscan informaciones a partir de un proceso recordatorio, algunos datos pueden no representar con precisión los acontecimientos del pasado. Sin embargo, se constituye la mejor forma de conocer los aspectos significativos y con cierta persistencia temporal en la trayectoria de los individuos y que no afecta la visión global de los aspectos relevantes de la formación.

\section{APLICACIONES PRÁCTICAS}

La información sobre la formación deportiva de jugadores, obtenida a través del IAFDV, permite a los responsables del desarrollo deportivo, como padres, entrenadores y dirigentes, la mejor orientación de sus acciones y programas, para asegurar una formación deportiva adequada. Investigaciones sobre las actividades, relaciones interpersonales y contextos de práctica deportiva con el IAFDV en jugadores de élite de voleibol, por exemplo, puede identificar el camino recorrido por estos jugadores y facilitar la formación deportiva cada vez más amplia y cualificada de jugadores de alto rendimiento, así como practicantes de deportes a lo largo de la vida.

Agradecimiento para la Coordenação de Aperfeiçoamento de Pessoal de Nível Superior (CAPES) de Brasil por la concesión de becas Capes DS e PDSE.

\section{REFERENCIAS}

1. Aiken, L. (1985). Three coeffients for analyzing the reability and validity of ratings. Educational and Psychological Measurement, 45, 131-143.

2. Ato, M., López, J. J. y Benavente, A. (2013). Un sistema de clasificación de los diseños de investigación en psicología. Anales de Psicología, 29(3), 1038-1059. doi:10.6018/analesps.29.3.178511.

3. Balish, S. y Côté, J. (2013). The influence of community on athletic development: an integrated case study. Qualitative Research in Sport, Exercise and Health, 6(1), 98-120. doi:10.1080/2159676X.2013.766815.

4. Blázquez-Manzano, A. (2011). Diseño y validación de un cuestionario para analizar la calidad en empleados de servicios deportivos públicos de las mancomunidades de municipios extremeñas. e-balonmano.com: Revista de Ciencias del Deporte, 7(3), 181-192

5. Bohórquez Gómez-Millán, M. R. y Checa Esquiva, I. (2017). Diseño y validez de contenido de una entrevista para la evaluación psicológica de porteros de fútbol. Revista de Psicología 
Collet, C., Nascimento, J. V., Folle, A., Ibáñez, S. J.

Aplicada al Deporte y el Ejercicio Físico, 2(1), e3, 1-12. doi:10.5093/rpadef2017a3

6. Carretero-Dios, H. y Pérez, C. (2005). Normas para el desarrollo y revisión de estudios instrumentales. International Journal of Clinical and Health Psychology, 5, 521-551.

7. Cassepp-Borges, V., Balbinotti, M. A. A. y Teodoro, M. L. M. (2009). Tradução e validação de conteúdo: uma proposta para a adaptação de instrumentos. In L. Pasquali (Ed.), Instrumentação Psicológica: fundamentos $\mathrm{e}$ práticas (pp. 506-520). Porto Alegre: ARTMED.

8. Castejón-Oliva, Santos-Pastor y Palacios-Picos. (2015). Cuestionario sobre metodología y evaluación en formación inicial en Educación Física. Revista Internacional de Medicina y Ciencias de la Actividad Física y el Deporte, 15(58), 245-267. doi:10.15366/rimcafd2015.58.004.

9. Collet, C., Nascimento, J. V., Ramos, V. y Stefanello, J. M. F. (2011). Construção e validação do instrumento de avaliação do desempenho técnico-tático no voleibol. Revista Brasileira de Cineantropometria e Desempenho Humano, 13(1), 43-51. doi:10.5007/1980$0037.2011 \mathrm{v} 13 \mathrm{n} 1 \mathrm{p} 43$.

10. Collet, C., Nascimento, J. V., Folle, A., \& Ibáñez, S. J. (2017). Atividades de prática deliberada e jogo deliberado na formação esportiva de atletas de elite do voleibol: diferenças entre os sexos. e-balonmano.com: Revista de Ciencias del Deporte, 13(2), 95-104.

11. Cordente, D., González-Víllora, S., PastorVicedo, J. C. y Contreras, O. (2018). Diseño y validación de un cuestionario para valorar las actitudes de los escolares hacia la discapacidad visual. Cuadernos de Psicología del Deporte, 18(1), 123-132.

12. Côté, J. (1999). The influence of the family in the development of talent in Sport. The Sport Psychologist, 13, 395-417.

13. Côté, J., Ericsson, K. A. y Law, M. P. (2005). Tracing the development of athletes using retrospective interview methods: a proposed interview and validation procedure for reported information. Journal of Applied Sport
Psichology, 17(1), 1-19. doi:10.1080/10413200590907531.

14. Côté, J. y Hay, J. (2002). Children's involvement in sport: a developmental perspective. In J. M. Silva y D. Stevens (Eds.), Psychological foundations of sport (pp. 503-519). Boston, MA: Allyn and Bacon.

15. Côté, J., Lidor, R. y Hackfort, D. (2009). To sample or to specialize? Seven postulates about youth sport activities and elite performance. International Journal of Sport and Exercise Psychology, 9, 7-17.

16. Côté, J., Turnnidge, J. y Vierimaa, M. (2016). A personal assets approach to youth sport. In $\mathrm{K}$. Green y A. Smith (Eds.), Routledge handbook of youth sport. (pp. 243-255). London and New York: Routledge.

17. Côté, J. y Vierimaa, M. (2014). The developmental model of sport participation: 15 years after its first conceptualization. Science \& Sports, 29(Supl.), 63-69. doi:http://dx.doi.org/10.1016/j.scispo.2014.08.13 3

18. Coutinho, P. A. d. S. (2014). O percurso para a excelência no desporto: estudo retrospectivo de fatores de treino e psicossociais em voleibolistas portugueses. (Doutoramento em Ciências do Desporto - Tese de doutorado), Universidade do Porto, Porto.

19. Crewson, P. Applied statistics desktop reference guide. Recuperated from http://www.acastat.com/statbook/statbook.html

20. Cronbach, L. J. (1951). Coefficient Alpha and the internal structure of tests. Psychometrika, 16(3), 297-334.

21. Cronin, L. D. y Allen, J. (2017). Development and initial validation of the Life Skills Scale for Sport. Psychology of Sport and Exercise, 28, 105-119. doi:http://dx.doi.org/10.1016/j.psychsport.2016.1 1.001 .

22. Drost, E. A. (2011). Validity and reliability in social science research. Education Research and Perspectives, 38(1), 105-123.

23. Ericsson, K. A., Krampe, R. T. y Tesch-Romer, C. (1993). The role of deliberate practice in the 


\section{Construcción y validación de un instrumento para el análisis de la formación deportiva en voleibol}

acquisition of expert performance. Psychological Review, 100(3), 363-406.

24. Escobar-Pérez, J. y Cuervo-Martínez, Á. (2008). Validez de contenido y juicio de expertos: Una aproximación a su utilización. Avances en Medición, 6, 27-36.

25. Escurra, L. M. (1988). Cuantificación de la validez de contenido por criterio de jueces. Revista de Psicología, 6, 103-111.

26. Flintoff, A., Foster, R. y Wystawnoha, S. (2011). Promoting and sustaining high quality physical education and school sport through school sport partnerships. European Physical Education Review, 17(3), 341-351. doi:10.1177/1356336x11416731

27. Folle, A., Quinaud, R. T., Barroso, M. L. C., Rocha, J. C. S., Ramos, V. y Nascimento, J. V. (2014). Construção e validação preliminar de instrumento de avaliação do desempenho técnico-tático individual no basquetebol. Journal of Physical Educationa, 25(3), 405-418. doi:10.4025/reveducfis.v25i3.23085.

28. Fraser-Thomas, J., Strachan, L. y Jeffery-Tosoni, S. (2013). Family influence on children's involvement in sport. In J. Côté y R. Lidor (Eds.), Conditions of children's talent development in sport (pp. 179-196). Morgantown: Copyright.

29. Gamonales, J. M., León, K., Muñoz, J., González, S. y Ibáñez, S. J. (2018). Validación del IOLF5C para la eficacia del lanzamiento en fútbol para ciegos. Revista Internacional de Medicina y Ciencias de la Actividad Física y el Deporte, 18(70), 361-381. doi:http://dx.doi.org/10.15366/rimcafd2018.70.0 10.

30. García-López, L. M., González-Víllora, S., Gutiérrez, D. y Serra, J. (2013). Development and validation of the game performance evaluation tool (GPET) in soccer. SportK. Revista Euroamericana de Ciencias del Deporte, 2(1), 89-99. doi:10.15366/rimcafd2016.63.008.

31. García-Martín, A., Antúnez, A., e Ibáñez, S. J. (2016). Análisis del proceso formativo en jugadores expertos: validación de instrumento. Revista Internacional de Medicina y Ciencias de la Actividad Física y del Deporte, 16(61), 157182. doi:10.15366/rimcafd2016.61.012.

32. García-Santos, D., e Ibáñez, S. J. (2016). Diseño y validación de un instrumento de observación para la valoración de un árbitro de baloncesto (IOVAB). Sport TK: Revista Euroamericana de Ciencias del Deporte, 5(2), 15-26.

33. Gómez-Carmona, P. M., Cervera, V. y Benito, P. J. (2014). Diseño y validación de un cuestionario socio-emocional para jóvenes futbolistas de élite. Revista Internacional de Medicina y Ciencias de la Actividad Física y del Deporte, 14(55), 10-15.

34. González, S., Ibáñez, S. J., Feu, S. y Galatti, L. R. (2017). Programas de intervención para la enseñanza deportiva en el contexto escolar, PETB y PEAB: Estudio preliminar. Retos, 31, 107-113.

35. Hernández-Sampieri, R., Fernández-Collado, C. y Baptista-Lucio, M. P. (2014). Metodología de la Investigación (6 ed.). México: Mc Graw Hill Education.

36. Hyrkäs, K., Appelqvist-Schmidlechner, K. y Oksa, L. (2003). Validating an instrument for clinical supervision using an expert panel. International Journal of Nursing Studies, 40(6), 619-625.

37. Holt, N. L. y Neely, K. C. (2011). Positive Youth Development Through sport: A review. Revista Iberoamericana de Psicología del Ejercicio y el Deporte, 6(2), 299-316.

38. Keegan, R. J., Harwood, C. G., Spray, C. M. y Lavallee, D. E. (2009). A qualitative investigation exploring the motivational climate in early career sports participants: coach, parent and peer influences on sport motivation. Psychology of Sport and Exercise, 10, 361-372. doi:10.1016/j.psychsport.2008.12.003.

39. Latorre, P. Á. y Pantoja, A. (2013). Diseño y validación de un cuestionario de propensión al accidente deportivo (PAD-22). Cuadernos de Psicología del Deporte, 13(1), 51-62.

40. Lima, E. P., Vasconcelos, A. G., Berger, W., Kristensen, C. H., Nascimento, E. D., Figueira, I. y Mendlowicz, M. V. (2016). Cross-cultural adaptation of the Posttraumatic Stress Disorder Checklist 5 (PCL-5) and Life Events Checklist 5 
Collet, C., Nascimento, J. V., Folle, A., Ibáñez, S. J.

(LEC-5) for the Brazilian context. Trends Psychiatry Psychother, 38(4), 207-215. doi:10.1590/2237-6089-2015-0074.

41. Lucena-Santos, P., Carvalho, S. A., Oliveira, M. d. S. y Pinto-Gouveia, J. (2017). Body-Image Acceptance and Action Questionnaire: Its deleterious influence on binge eating and psychometric validation. International Journal of Clinical and Health Psychology, 17(2), 151-160. doi:10.1016/j.ijchp.2017.03.001.

42. Lynn, M. R. (1986). Determination and quantification of content validity. Nursing Research, 35(6), 382-386.

43. MacDonald, D. y Baker, J. (2013). Circumstantial development: Birthdate and birthplace effects on athlete development. In J. Côté y R. Lidor (Eds.), Conditions of Children's Talent Development in Sport (pp. 197-208). Morgantown: Fitness Information Technology.

44. Manchola-González, J., Bagur-Calafat, C. y Girabent-Farrés, M. (2017). Fiabilidad de la versión española del cuestionario de actividad física PAQ-C. Revista Internacional de Medicina y Ciencias de la Actividad Física y del Deporte, $17(65)$, 139-152. doi:10.15366/rimcafd2017.65.008.

45. Marques, R. F. R., Lima, C. P., Moraes, C., Nunomura, M. y Simões, E. C. (2014). Formação de jogadores profissionais de voleibol: relações entre atletas de elite e a especialização precoce. Revista Brasileira de Educação Física e Esporte, São Paulo, 28(2), 293-304. doi: http://dx.doi.org/10.1590/180755092014000200293.

46. Martínez-Abellán, A., Conesa-Ros, E., MoránNavarro, R., Pallarés, J. G., Cruz-Sánchez, E., Ortega, E. y López-Gullón, J. M. (2016). Diseño $\mathrm{y}$ validación de contenido de un cuestionario sobre Pérdida de Peso en Deportes de Combate. Cuadernos de Psicología del Deporte, 16(3), 113122.

47. Mendo, A. H. (2001). Un cuestionario para evaluar la calidad en programas de actividad física. Revista de Psicología del Deporte, 10(2), 179-196

48. Merino, C. S. y José Livia, J. S. (2009). Intervalos de confianza asimétricos para el índice la validez de contenido: Un programa Visual Basic para la V de Aiken. Anales de Psicología, 25(1), 169-171.

49. Montero, I. y León, O. G. (2007). A guide for naming research studies in Psychology. International Journal of Clinical and Health Psychology, 7(3), 847-862.

50. Moreno, J. A., López, M., Martínez-Galindo, C. M., Alonso, N. y González-Cutre, D. (2007). Validación preliminar de la escala de percepción del clima motivacional de los iguales (CMI) y la escala de las orientaciones de meta en el ejercicio (GOES) con practicantes españoles de actividades físico-deportivas. Revista Iberoamericana de Psicología del Ejercicio y el Deporte, 1(2), 13-28.

51. Ortega, E., Jiménez, J. M., Palao, J. M. y Sáinz de Barranda, P. (2008). Diseño y validación de un cuestionario para valorar las preferencias y satisfacciones en jóvenes jugadores de baloncesto. Cuadernos de Psicología del Deporte, $8(2), 39-58$.

52. Pazo, C. I., Sáenz-López, P., \& Fradua, L. (2012). Influencia del contexto deportivo en la formación de los futbolistas de la selección española de fútbol. Revista de Psicologia del Deporte, 21(2), 291-299.

53. Penfield, R. D. y Giacobbi, P. R. (2004). Applying a Score Confidence Interval to Aiken's Item Content-Relevance Index. Measurement in Physical Education and Exercise Science, 8(4), 213-225.

54. Polit, D. F. y Beck, C. T. (2003). Nursing Research: Principles and Methods ( 7 ed.). Philadelphia: Lippincott Williams \& Wilkins.

55. Prieto, G. y Delgado, A. R. (2010). Fiabilidad y Validez. Papeles del Psicólogo, 31(1), 67-74.

56. Robles-Rodríguez, A., Robles-Rodríguez, J., Giménez-Fuentes-Guerra, F. J. y Abad-Robles, M. T. (2016). Validación de una entrevista para estudiar el proceso formativo de judokas de élite. Revista Internacional de Medicina y Ciencias de la Actividad Física y del Deporte, 16(64), 723738. doi:10.15366/rimcafd2016.64.007.

57. Serra-Olivares, J. y García-López, L. M. (2016). Diseño y validación del test de conocimiento 


\section{Construcción y validación de un instrumento para el análisis de la formación deportiva en voleibol}

táctico ofensivo en fútbol (TCTOF). Revista Internacional de Medicina y Ciencias de la Actividad Física y del Deporte/International Journal of Medicine and Science of Physical Activity and Sport, 16(63). doi:10.15366/rimcafd2016.63.008.

58. Skjong, R. y Wentworth, B. H. (2001). Expert judgment and risk perception. Paper presented at the The Eleventh International Offshore and Polar Engineering Conference, Stavanger, Norway.

59. Villarejo, D., Ortega, E., Gómez, M.-Á. y Palao, J.-M. (2014). Design, validation, and reliability of an observational instrument for ball possessions in rugby union. International Journal of Performance Analysis in Sport, 14(3), 955967.

60. Voutilainen, P. y Liukkonen, A. (1995). Senior Monitor - laadun arviointimittarin sisällön validiteetin määrittäminen. Hoitotiede, 1, 51-56.

61. Weiss, M. R., Bolter, N. D. y Kipp, L. E. (2014). Assessing impact of physical activity-based youth development programs: validation of the Life Skills Transfer Survey (LSTS). Research Quarterly for Exercise and Sport, 85(3), 263-278. doi:10.1080/02701367.2014.931558. 